\title{
Synthesis of BMS-663068
}

Key words

BMS-663068

HIV attachment inhibitors<smiles>[13CH3]NCC1(c2ccn(S(=O)(=O)O)c2)OCCO1</smiles>

$\mathrm{mp} 188^{\circ} \mathrm{C}$<smiles>[13CH3]N1CC(=O)c2ccn(-c3ccccc3)c2C1</smiles>
p $168^{\circ} \mathrm{C}$
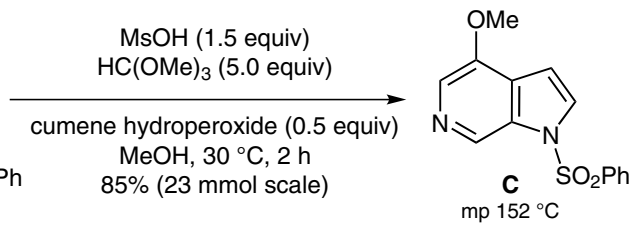

$\mathrm{H}_{2} \mathrm{O}_{2}$ (2.0 equiv) $\mathrm{MeReO}_{3}$ (0.02 equiv) $\mathrm{CH}_{2} \mathrm{Cl}_{2}$, r.t., $24 \mathrm{~h}$ (35 mmol scale)<smiles>COc1cnc(Br)c2[nH]c(Br)c(C(=O)C(=O)O)c12</smiles>

$\mathbf{F}$
$\mathrm{MeO}_{2} \mathrm{C}-\mathrm{COCl}$ (2.0 equiv) $\mathrm{AlCl}_{3}$ (4.3 equiv) $\mathrm{CH}_{2} \mathrm{Cl}_{2}-\mathrm{MeNO}_{2}, 0{ }^{\circ} \mathrm{C}, 5 \mathrm{~h}$;

then $\mathrm{NaOH}$ (3.5 equiv) $\mathrm{THF}-\mathrm{H}_{2} \mathrm{O}$, r.t.; then $\mathrm{HBr}$ $79 \%$ (6.5 mmol scale)<smiles>COc1cnc(Br)c2[nH]ccc12</smiles>

. $\mathrm{HCl} \cdot \mathrm{H}_{2} \mathrm{O}$
E $\mathrm{mp} 160^{\circ} \mathrm{C}$ (dec)

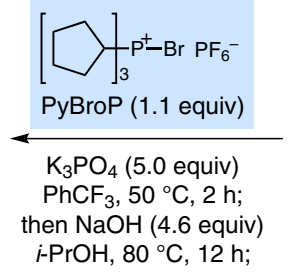<smiles>COc1c[n+]([O-])cc2c1ccn2-c1ccccc1</smiles>
then $\mathrm{HCl}, i-\mathrm{PrOH}, 50^{\circ} \mathrm{C}$

$70 \%$ from C

6-azaindoles

Ullmann-GoldbergBuchwald coupling

bromination<smiles>O=C(c1ccccc1)N1CCN(Cl)C1</smiles>

CDI (1.7 equiv) DMF, $10^{\circ} \mathrm{C}, 3 \mathrm{~h}$

then add $\mathbf{G}$ (1.3 equiv) r.t. $2 \mathrm{~h}$ $85 \%$ (13 mmol scale)<smiles>COc1cnc(Br)c2[nH]cc(C(=O)C(=O)N3CCN(C(=O)c4ccccc4)CC3)c12</smiles>
$\mathbf{H}$
$\mathrm{mp} 252.5^{\circ} \mathrm{C}$

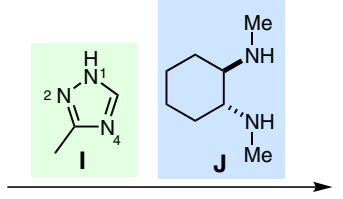

I (1.5 equiv) $\mathbf{J}$ (1.5 equiv) Cul (0.2 equiv) $\mathrm{KOH}$ (2.0 equiv) $\mathrm{MeCN}, 75^{\circ} \mathrm{C}, 18 \mathrm{~h}$ then $\operatorname{LiBr}$ ( 1.1 equiv) $66 \%$ (23 mmol scale)

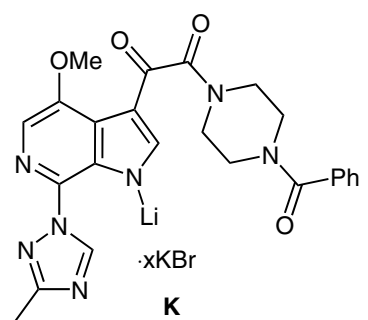

$\mathrm{mp}$ not reported
Significance: Attachment inhibitor BMS-663068 is currently in clinical development for the treatment of HIV infection. Key steps in the synthesis depicted are (1) a radical-mediated redox-aromatization to generate the 6-azaindole $(\mathbf{B} \rightarrow \mathbf{C})$ and (2) the regioselective bromination of an $\mathrm{N}$-oxide using PyBroP $(\mathbf{D} \rightarrow \mathbf{E})$.
Comment: High regioselectivity was observed in the copper(I)-mediated UIImann-GoldbergBuchwald coupling $(\mathbf{H} \rightarrow \mathbf{K})$ using the diamine ligand $\mathbf{J}(\mathrm{N} 1 / \mathrm{N} 2=22: 1)$, whereas a thermal $\mathrm{S}_{N} \mathrm{Ar}$ reaction gave $\mathrm{N} 1 / \mathrm{N} 2=1: 1$. Alternative conditions for the bromination of the $\mathrm{N}$-oxide $\mathbf{D}$ led mainly to deoxygenation.

SYNFACTS Contributors: Philip Kocienski

Synfacts 2014, 10(12), 1239 Published online: 18.11.2014

Dol: 10.1055/s-0034-1379407; Reg-No.: K05514SF 